\title{
Analyzing and tuning emission characteristics of top-emitting organic light-emitting devices
}

\author{
Chun-Liang Lin \\ Ping-Yuan Hsieh \\ Huo-Hsien Chiang \\ Chung-Chih Wu
}

\begin{abstract}
Top-emitting organic light-emitting devices (OLEDs) have several technical merits for application in active-matrix OLED displays. Generally, stronger microcavity effects inherent with topemitting OLEDs, however, complicate the optimization of device efficiency and other viewing characteristics, such as color and viewing-angle characteristics. In this paper, using the rigorous classical electromagnetic model based on oscillating electric dipoles embedded in layered structures, the emission characteristics of top-emitting OLEDs as a function of device structures will be analyzed. From comprehensive analysis, trends in the dependence of ewmission characteristics on device structures were extracted, and, accordingly, a general methodology for optimizing viewing characteristics of top-emitting OLEDs for display applications will be suggested. The effectiveness of the analysis and the methodology was confirmed by experimental results.
\end{abstract}

Keywords — Organic light-emitting devices, top-emitting organic light-emitting devices, microcavity effects.

\section{Introduction}

Top-emitting organic light-emitting devices (OLEDs) that render feasible fabrication of OLED displays on opaque substrates such as $\mathrm{Si}$ wafers and active-matrix backplanes with complicated pixel circuits of thin-film transistors have been the subject of intensive studies recently. ${ }^{1-8}$ Top-emitting OLEDs, in general, are composed of a reflective bottom contact, organic layers, and a (semi)transparent top contact for light out-coupling. The (semi)transparent top contact usually consists of transparent conducting oxides, thin metal films, or their combinations. These top contacts, in general, show a rather strong reflection due to large refractive-index mismatches with the organic layers below and air above, leading to strong microcavity effects on the emission characteristics of devices. ${ }^{5-8}$ Strong microcavity effects usually lead to a strong dependence of the out-coupling efficiency and color on device structures, and a large variation of color and brightness with viewing angles ${ }^{5-10}$ It is generally found that efficiency, color, and viewing-angle characteristics (both color and intensity) of top-emitting OLEDs interplay with the optical structures of devices in such a subtle way that makes simultaneous optimization of all these viewing characteristics for display applications through tuning device structures particularly challenging. ${ }^{5-9}$ A methodology that can effectively guide the optimization of the emission characteristics will thus be useful. In this paper, we present a systematic and comprehensive analysis of the emission characteristics of top-emitting OLEDs as a function of device structures, and based on these results a general methodology for optimizing viewing characteristics of top-emitting OLEDs for display applications is suggested.

\section{Device structure of the top-emitting OLEDs}

One working top-emitting OLED structure, ${ }^{8}$ which uses a reflective metal as the bottom anode and a semitransparent thin metal as the top cathode, was used as an example for analysis. In this case, Ag with a thin silver oxide induced at the surface is used as the bottom anode, which provides a hole-injection capability competitive with the usual indium tin oxide anode and a high reflectivity of $82-91 \%$ over the visible range. ${ }^{8}$ The organic multilayer structure on top of the anode in sequence consists of $4,4^{\prime}, 4^{\prime \prime}$-tris(3-methylphenylphenylamino)triphenylamine (m-MTDATA) as the hole-injection layer, ${ }^{11} \alpha$-naphthylphenylbiphenyl diamine $(\alpha-\mathrm{NPD}$, fixed at $20 \mathrm{~nm})$ as the hole-transport layer, ${ }^{12}$ tris(8-hydroxyquinoline) aluminum $\left(\mathrm{Alq}_{3}\right)$ as the electrontransport and emitting layer. ${ }^{12}$ The cathode is composed of multiple functional layers to achieve both optical transmission and effective electron injection. Ultrathin layers of $\mathrm{LiF}$ $(0.5 \mathrm{~nm})$ and $\mathrm{Al}(1 \mathrm{~nm})$ are deposited in sequence as the electron-injecting contact. ${ }^{3} \mathrm{~A}$ thin layer $(20 \mathrm{~nm})$ of $\mathrm{Ag}$, which has relatively low optical absorption and the highest conductivity among all metals, then overlays the $\mathrm{LiF} / \mathrm{Al}$ contact for reducing sheet resistance of the composite cathode. ${ }^{3}$ The multilayer cathode is further capped with a high-refractive-index dielectric layer of thermally evaporated $\mathrm{TeO}_{2}(n \sim 2.1-2.3)$ to modulate the optical properties (e.g., transmission, reflection and absorption) of the composite cathode composing thin metal/dielectric capping. $3,5,7,8$ The thicknesses of organic layers ( $m$-MTDATA, $\mathrm{Alq}_{3}$ ) and the $\mathrm{TeO}_{2}$ layer were varied in the analysis. The complex refractive indices of the material layers required for the calculation were obtained by spectroscopic ellipsometry.

Revised version of a paper presented at the 2004 SID International Symposium held May 25-27, 2004, in Seattle, Washington.

The authors are with the Department of Electrical Engineering, Graduate Institute of Electro-Optical Engineering, National Taiwan University, No. 1, Sec. 4, Roosevelt Rd., Taipei, Taiwan, 10617; telephone +886-2-2367-7467, fax +886-2-2363-5251 x346; e-mail: chungwu@cc.ee.ntu.edu.tw.

(c) Copyright 2005 Society for Information Display 1071-0922/05/1302-0253\$1.00 

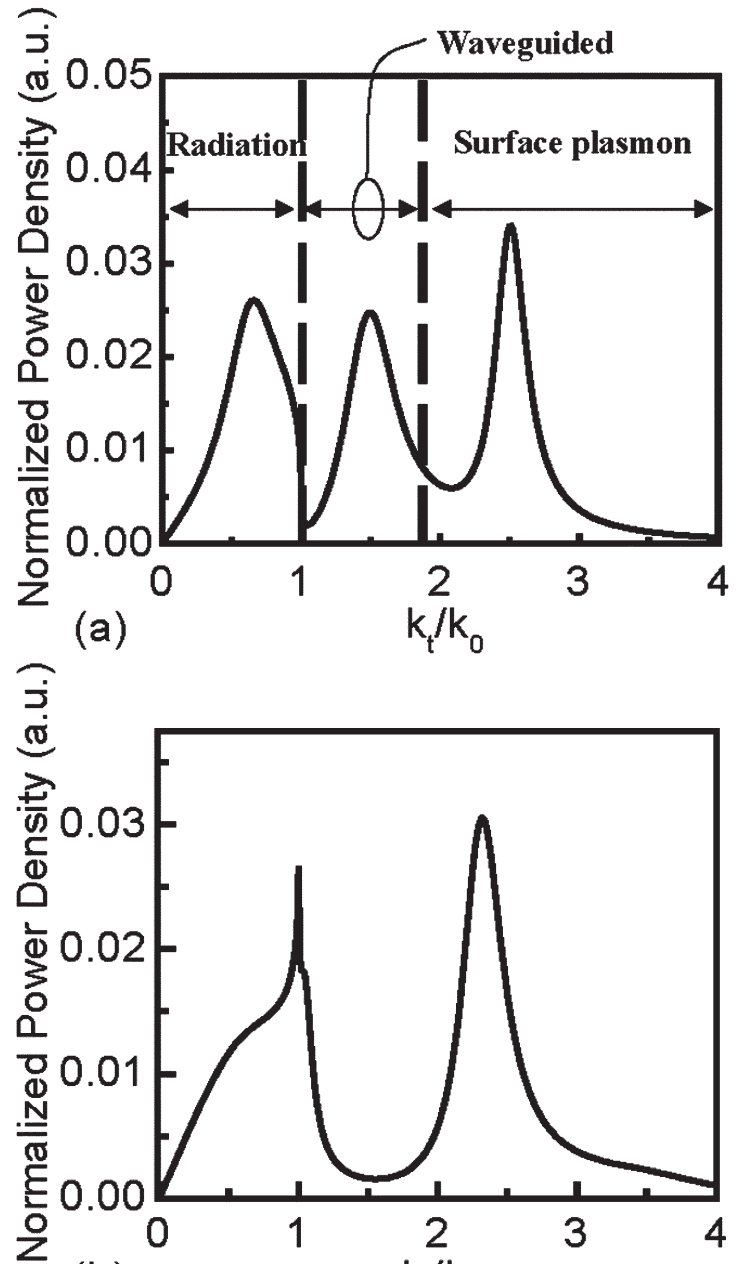

(b)

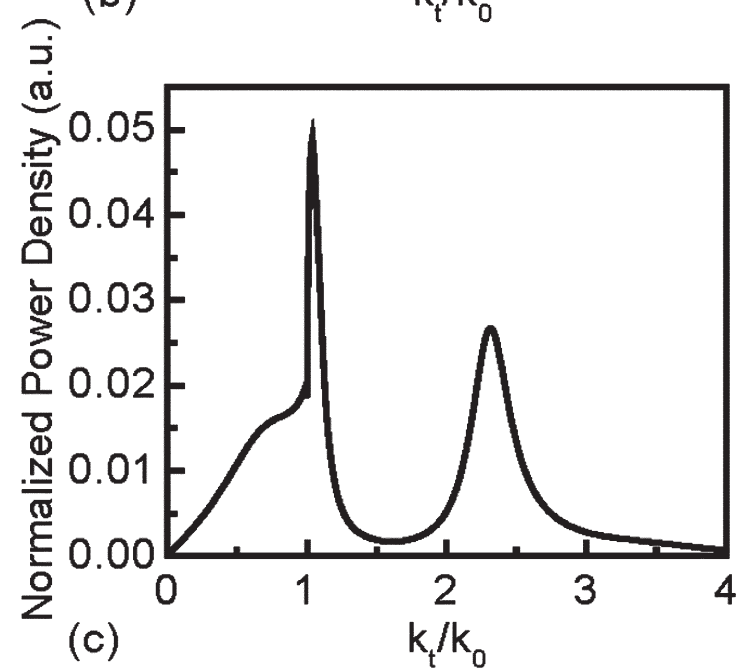

FIGURE 1 - Calculated normalized power density as a function of normalized in-plane wave vector $k_{t} / k_{0}$ for devices with (a) $\left(\mathrm{TeO}_{2}, \mathrm{Alq}_{3}\right.$, $m$-MTDATA $)=(0,60$, and $30 \mathrm{~nm}),\left(\right.$ b) $\left(\mathrm{TeO}_{2}, \mathrm{Alq}_{3}, m-\mathrm{MTDATA}\right)=(40$, 60 , and $30 \mathrm{~nm})$, and $(\mathrm{c})\left(\mathrm{TeO}_{2}, \mathrm{Alq}_{3}, m-\mathrm{MTDATA}\right)=(40,65$, and $35 \mathrm{~nm})$ at a single emission wavelength of $550 \mathrm{~nm}$.

\section{Optical modeling of top-emitting OLEDs}

The optical model used for performing the analysis adopts a classical approach based on the equivalence between the emission of a photon due to an electrical dipole transition and the radiation from a classical electrical dipole antenna, which can take into account loss due to electrodes and has been described in previous literature. ${ }^{13-16}$ With plane-wave expansion of the dipole field, the full-vectorial electromagnetic fields generated by a radiation dipole embedded in a layered structure is calculated, from which the distribution of the radiation power into different plane-wave modes and the far-field radiation related to emission characteristics of an OLED are obtained. ${ }^{13-16}$ In the plane-wave expansion, each plane-wave mode can be characterized by an in-plane wave vector $k_{t}$, where $k_{t}$ is the component of the wave vector parallel to the layer surface. To model emission characteristics of an OLED, it is assumed that the emitting layer contains an ensemble of mutually incoherent dipole radiators with distributions in dipole orientations (a random isotropic distribution), locations (a decaying exponential distribution from the $\alpha-\mathrm{NPD} / \mathrm{Al}_{3}$ interface into $\mathrm{Al}_{3}$ with an exciton diffusion length of $10 \mathrm{~nm}),{ }^{17}$ and frequencies [using the photoluminescence (PL) spectrum of $\mathrm{Alq}_{3}$ as the intrinsic spectral distribution of the dipole radiators]. The total emission intensity $I$ from the OLED as a function of the wavelength $\lambda$ and the viewing angle $\theta[i . e ., I(\theta, \lambda)$, with $\theta$ measured relative to the surface normal] is then obtained by averaging contributions over these distributions. Color coordinates of emission at various angles $\left[u^{\prime}(\theta), v^{\prime}(\theta)\right]$ can then be calculated from $I(\theta, \lambda)$. To better quantify differences in colors, the $1976 u^{\prime} v^{\prime}$ uniform color space was used. ${ }^{18}$

In the present case, three device parameters were varied in analyzing the emission characteristics: (1) the distance of the emitting dipole to the bottom contact (through varying the $m$-MTDATA thickness); (2) the distance of the emitting dipole to the top contact (through varying the $\mathrm{Al}_{3}$ thickness); and (3) the optical properties of the top contact (through varying the $\mathrm{TeO}_{2}$ thickness). To quantify their effects on emission characteristics, four parameters are defined and calculated: (1) the out-coupling efficiency which is the ratio of out-coupled emission to internally generated emission; (2) the saturation offset which is defined as $\left[u^{\prime}\left(0^{\circ}\right)-u^{\prime}(520 \mathrm{~nm})\right]^{2}+\left[v^{\prime}\left(0^{\circ}\right)-v^{\prime}(520 \mathrm{~nm})\right]^{2}$ and measures the color difference between the $0^{\circ}$ emission and a target saturated color (e.g., $520 \mathrm{~nm}$ monochromatic green light here); (3) the color shift which is defined as [variance of $u^{\prime}(\theta)+$ variance of $\left.v^{\prime}(\theta)\right]$ and measures variation of colors over angles; and (4) the Lambertian offset which is defined as $\Sigma\left|I(\theta) / I\left(0^{\circ}\right)-\cos \theta\right|$ and measures the difference between the emission pattern and the ideal Lambertian distribution [where $I(\theta)$ is spectrally integrated $I(\theta, \lambda)]$.

\section{Analysis results of top-emitting OLEDs}

To acquire a better understanding of the dependence of emission mechanism on device structures of top-emitting OLEDs, the distribution of radiation power coupled into different plane-wave modes (represented by the in-plane wave vector $k_{t}$ ) is calculated for various device structures. Figure 1 shows the normalized power density at a single 

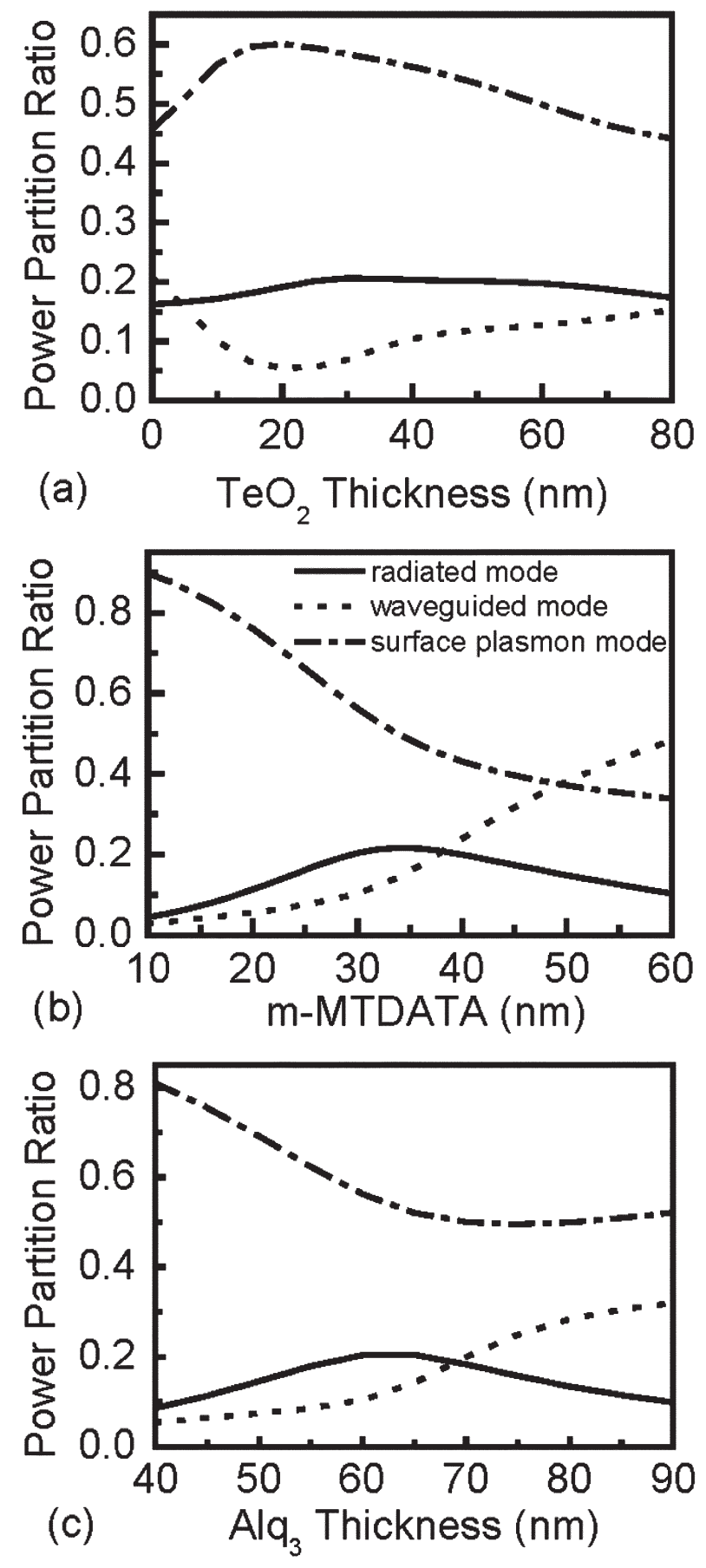

FIGURE 2 - Calculated partition ratios of power coupled into the three different modes (at a single emission wavelength of $550 \mathrm{~nm}$ ) as a function of (a) the $\mathrm{TeO}_{2}$ thickness [with $\left(\mathrm{Alq}_{3}\right.$, m-MTDATA) fixed at (60 and 30 $\mathrm{nm})$ ], (b) the m-MTDATA thickness [with $\left(\mathrm{TeO}_{2}, \mathrm{Alq}_{3}\right)$ fixed at (40 and $60 \mathrm{~nm})$ ], and (c) the $\mathrm{Alq}_{3}$ thickness [with $\left(\mathrm{TeO}_{2}, \mathrm{~m}-\mathrm{MTDATA}\right)$ fixed at (40 and $30 \mathrm{~nm})]$.

emission wavelength of $550 \mathrm{~nm}$ as a function of the in-plane wave vector $k_{t}$ (normalized to the free-space wave vector $k_{0}$ ) for three representative device structures: (a) $\left(\mathrm{TeO}_{2}, \mathrm{Al}_{4}\right.$, $m$-MTDATA $)=(0,60$, and $30 \mathrm{~nm}),(\mathrm{b})\left(\mathrm{TeO}_{2}, \mathrm{Alq}_{3}, m\right.$ MTDATA $)=(40,60$, and $30 \mathrm{~nm})$, and $(\mathrm{c})\left(\mathrm{TeO}_{2}, \mathrm{Alq}_{3}, \mathrm{~m}-\right.$ MTDATA $)=(40,65$, and $35 \mathrm{~nm})$. In these cases, modes with the in-plane wave vector $k_{t}$ of $0<k_{t}<k_{0}$ are associated with
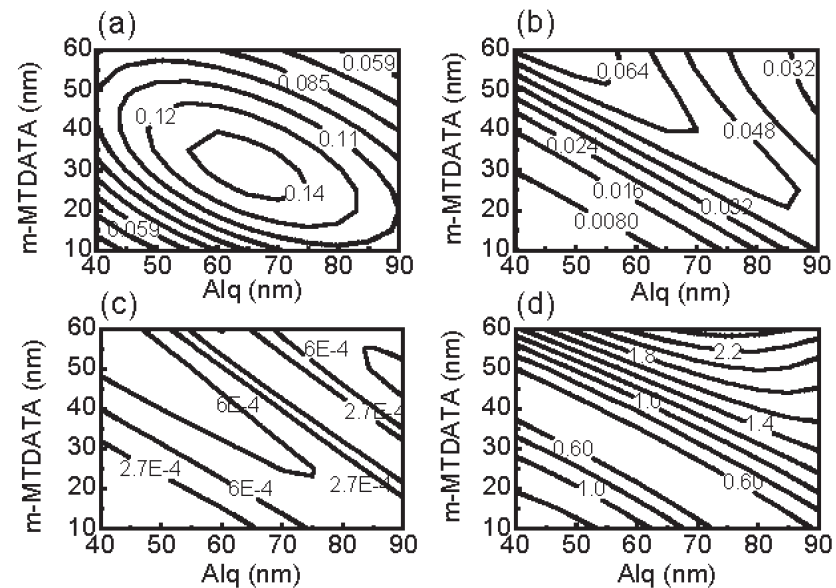

FIGURE 3 - Calculated contour plots of (a) out-coupling efficiency, (b) saturation offset, (c) color shift, and (d) Lambertian offset as a function of the $\mathrm{Alq}_{3}$ thickness and the m-MTDATA thickness with the $\mathrm{TeO}_{2}$ thickness fixed at $20 \mathrm{~nm}$.

free radiation modes, modes with an in-plane wave vector $k_{t}$ of $k_{0}<k_{t}<n k_{0}$ (where $n$ is the refractive index of the organic material) are associated with waveguided modes, and modes with the in-plane wave vector $k_{t}$ of $n k_{0}<k_{t}<\infty$ are associated with surface plasmon modes. Figures $2(\mathrm{a})-2(\mathrm{c})$ show the calculated partition ratios of power coupled into the three different modes as a function of the $\mathrm{TeO}_{2}$ thickness [with $\left(\mathrm{Alq}_{3}, m\right.$-MTDATA) fixed at $(60$ and $\left.30 \mathrm{~nm})\right]$, the $m$-MTDATA thickness [with $\left(\mathrm{TeO}_{2}, \mathrm{Alq}_{3}\right)$ fixed at (40 and 60 $\mathrm{nm})$ ], and the $\mathrm{Alq}_{3}$ thickness [with $\left(\mathrm{TeO}_{2}, m\right.$-MTDATA) fixed at $(40$ and $30 \mathrm{~nm})]$. From Figs. 1 and 2(a), one observes that by adding a $\mathrm{TeO}_{2}$ capping layer substantially suppresses the waveguided modes. The suppression of waveguided modes, however, does not completely contribute to the enhancement of free radiation modes, since it could be accompanied by enhanced surface plasmon modes as well. Nevertheless, by carefully adjusting the $\mathrm{TeO}_{2}$ thickness, one still can obtain a net gain in free radiation modes (out-coupled emission). In Figs. 1(b)-1(c) and 2(b)-2(c), it is observed that ratios of power coupled into surface plasmon modes decrease as the emitting dipoles are moved away from the two metallic electrodes with an increased thickness of the organic layers, which, however, are accompanied with increased coupling into waveguided modes. The compromise of these two competing factors results into maximum out-coupling of emission at certain thicknesses. Finally, one notices that although there are no substrate modes in the top-emitting devices as in the bottom-emitting devices, the ratio of out-coupling emission does not show obvious enhancement. The corresponding increase in either surface plasmon modes or waveguided modes in top-emitting devices substantially cancels the benefits of removing substrate modes.

Figures 3-5 show the calculated contour plots of four emission parameters (out-coupling efficiency, color saturation, color shift, and Lambertian offset) of the present topemitting devices as a function of $m$-MTDATA and Alq 3 thicknesses for the $\mathrm{TeO}_{2}$ thickness fixed at 20,40, and $60 \mathrm{~nm}$, respectively. Indeed, in varying the $\mathrm{TeO}_{2}$ thickness, 

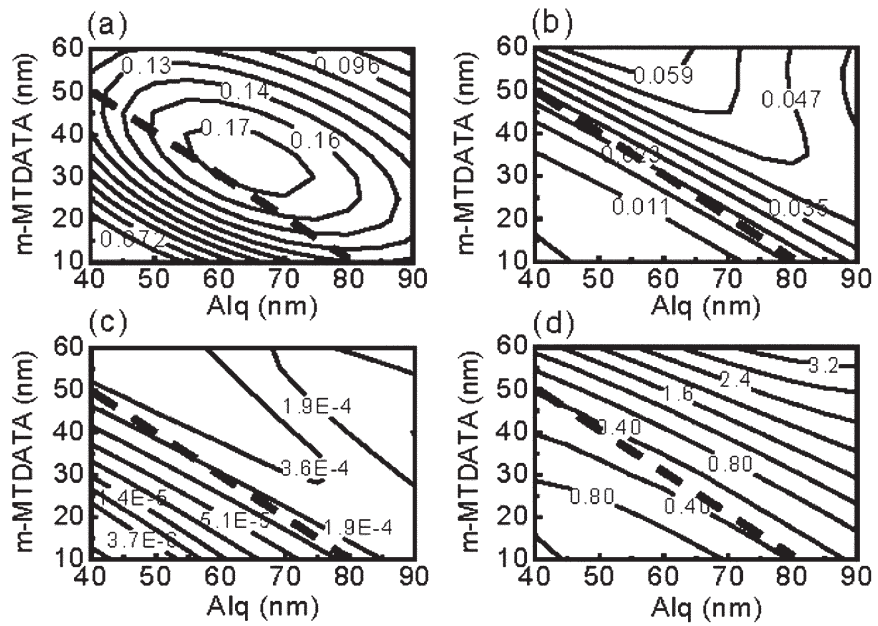

FIGURE 4 - Calculated contour plots of (a) out-coupling efficiency, (b) saturation offset, (c) color shift, and (d) Lambertian offset as a function of the $\mathrm{Alq}_{3}$ thickness and the m-MTDATA thickness with the $\mathrm{TeO}_{2}$ thickness fixed at $40 \mathrm{~nm}$. The thick dashed lines represent the condition of total $\mathrm{Alq}_{3}+m-$ MTDATA thickness of $90 \mathrm{~nm}$.

contours of all these characteristics remain similar. It indicates that the optical properties of the top contact affect the magnitudes of these characteristics but does not substantially alter their dependence on the thicknesses of organic layers. In all these figures, one observes that the contours of the out-coupling efficiency resemble concentric ellipses with the maximum near $\left(\operatorname{Alq}_{3}, m\right.$-MTDATA $)=(65$ and $35 \mathrm{~nm})$. Furthermore, near the efficiency maximum, the contours of saturation offset, color shift, and Lambertian offset resemble straight lines with a slope of $\sim-1$, which indicates that these viewing characteristics are roughly determined by the total thickness of organic layers (i.e., the cavity length) near the efficiency maximum. In general, the saturation offset and color shift decrease with the total thickness of organic layers. That is, color performances can be
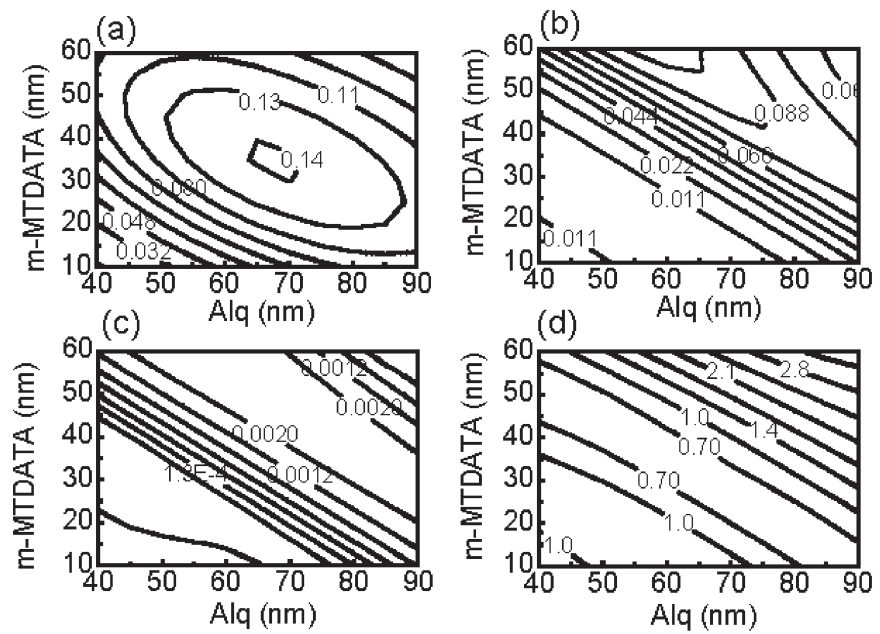

FIGURE 5 - Calculated contour plots of (a) out-coupling efficiency, (b) saturation offset, (c) color shift, and (d) Lambertian offset as a function of the $\mathrm{Alq}_{3}$ thickness and the m-MTDATA thickness with the $\mathrm{TeO}_{2}$ thickness fixed at $60 \mathrm{~nm}$. improved with a reduced cavity length near the efficiency maximum. On the other hand, contours of Lambertian offset show a minimum around the total $m$-MTDATA + Alq ${ }_{3}$ thickness of $80-100 \mathrm{~nm}$.

\section{$5 \quad$ Methodology for optimizing viewing characteristics of top-emitting OLEDs}

From results as shown in Figs. 3-5, in determining the optimal structure for a top-emitting OLED, it is plausible to first determine a cathode structure (i.e., $\mathrm{TeO}_{2}$ thickness in the present case) that would give the best overall device characteristics (including efficiency and other viewing characteristics), and then to fine tune the organic structure to achieve target viewing characteristics. In the course, one should be aware of the tradeoff between efficiency and other viewing characteristics.

As the first step of determining the cathode structure, an analysis as in Fig. 6, which shows calculated out-coupling efficiency, saturation offset, color shift, and Lambertian offset as a function of the $\mathrm{TeO}_{2}$ thickness [at a nearly optimalefficiency condition of $\left(\mathrm{Alq}_{3}, m\right.$-MTDATA $)=(60$ and 30 $\mathrm{nm}$ ) in this case], would be useful. From such analysis, one sees that a $\mathrm{TeO}_{2}$ thickness of $\sim 40 \mathrm{~nm}$ gives optimized overall characteristics. As a target for further design of devices, characteristics of a conventional bottom-emitting device (with the structure of glass/ITO $(120 \mathrm{~nm}) / \mathrm{m}$-MTDATA (30 $\left.\mathrm{nm}) / \alpha-\mathrm{NPD}(20 \mathrm{~nm}) / \mathrm{Al}_{3}(70 \mathrm{~nm}) / \mathrm{LiF} / \mathrm{Al}\right)$ are used as the reference. Measurements of such a device gave a saturation offset of $\sim 0.02$, a color shift of $\sim 1 \times 10^{-4}$, and a Lambertian offset of $\sim 0.13$. Calculation results as in Figs. 4 and 6 indicate that in general the top-emitting OLED may achieve color performances similar to or even better than those of a
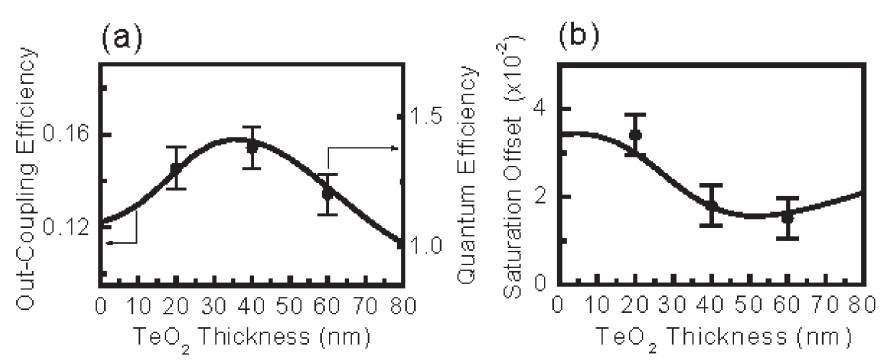

(c)
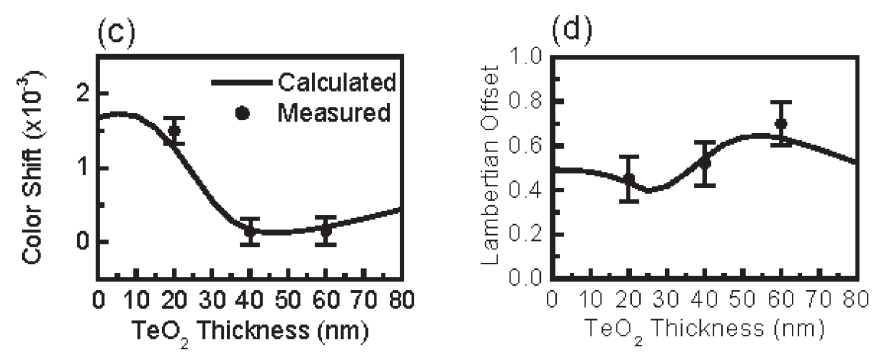

FIGURE 6 - Calculated values (lines) of (a) out-coupling efficiency, (b) saturation offset, (c) color shift, and (d) Lambertian offset as a function of the $\mathrm{TeO}_{2}$ thickness at the condition of $\left(\mathrm{Alq}_{3}, m-\mathrm{MTDATA}\right)=(60$ and $30 \mathrm{~nm}$ ). Corresponding results obtained from experiments (symbols) are also shown in each figure for comparison. 
bottom-emitting device with a slight tradeoff in efficiency, but cannot easily achieve the Lambertian offset of a bottomemitting device. By compromising efficiency and other viewing characteristics, a total $m$-MTDATA + $\mathrm{Alq}_{3}$ thickness of $90 \mathrm{~nm}$ could be picked to give viewing characteristics close to those of a bottom-emitting device. Such condition is represented by thick dashed lines in Fig. 4 (with a $\mathrm{TeO}_{2}$ thickness of $40 \mathrm{~nm}$ ). Along this line, varying the ratio of the $m$-MTDATA thickness to the Alq ${ }_{3}$ thickness does not alter color performance much, yet the efficiency is affected substantially with an optimal efficiency condition around $\left(\mathrm{Alq}_{3}\right.$, $m$-MTDATA $)=(60$ and $30 \mathrm{~nm})$. In summary, the device $($ device $\mathrm{B})$ with $\left(\mathrm{TeO}_{2}, \mathrm{Alq}_{3}, m\right.$-MTDATA $)=(40,60$, and 30 $\mathrm{nm}$ ) is expected to give acceptable overall characteristics. For comparison, a device (device A) with $\left(\mathrm{TeO}_{2}, \mathrm{Alq}_{3}, m\right.$ MTDATA $)=(40,65$, and $35 \mathrm{~nm})$ that is expected to give the highest efficiency, and a device (device $\mathrm{C}$ ) with $\left(\mathrm{TeO}_{2}, \mathrm{Alq}_{3}\right.$, $m$-MTDATA $)=(40,50$, and $30 \mathrm{~nm})$ that is expected to give even better color performances were also fabricated and tested.

\section{Experiment results}

To test the validity of the optimization methodology, a few test devices were fabricated and characterized. All the material layers in devices, including $\mathrm{TeO}_{2}$, were deposited by thermal evaporation within one vacuum pump-down in a multiple-source vacuum chamber. ${ }^{19,20}$ The fabricated devices were encapsulated with a cover glass under a dry-nitrogen atmosphere. The current-voltage-brightness $(I-V-L)$ characteristics of devices were measured with a source measurement unit and a Si photodiode calibrated with a Photo Research PR650 spectroradiometer. The electroluminescence (EL) spectra were taken with a calibrated CCD spectragraph.

Along with the PL spectrum of $\mathrm{Alq}_{3}$, Figs. 7(a)-(c) show measured (symbols) and simulated (solid lines) EL spectra with relative intensities at viewing angles of $0^{\circ}, 30^{\circ}$, and $60^{\circ}$ for devices with a fixed organic structure of ( $\mathrm{Alq}_{3}$, $m$-MTDATA $)=(60$ and $30 \mathrm{~nm})$ and the varied $\mathrm{TeO}_{2}$ thickness of 20,40 , and 60 , respectively. The measured spectra and relative intensities (symbols) are in excellent agreement with the calculated ones (solid lines), confirming the accuracy of the simulation. In Fig. 6, the four emission parameters extracted from the experimental results of the three devices (symbols) are compared with the calculated results (lines). Fairly good agreement is obtained, confirming the choice of $40 \mathrm{~nm}$ as the optimal $\mathrm{TeO}_{2}$ thickness. Figures $8(\mathrm{a})-(\mathrm{c})$ show measured (symbols) and simulated (solid lines) EL spectra with relative intensities at viewing angles of $0^{\circ}, 30^{\circ}$, and $60^{\circ}$ for test devices (A, B, and C). Figure $8(\mathrm{~d})$ also compares polar plots of the measured EL intensity (normalized to the $0^{\circ}$ intensity) for the three devices with the Lambertian distribution. Further, Fig. 9 shows measured external EL quantum efficiencies of the three devices vs. driving current. The quantum efficiencies of these
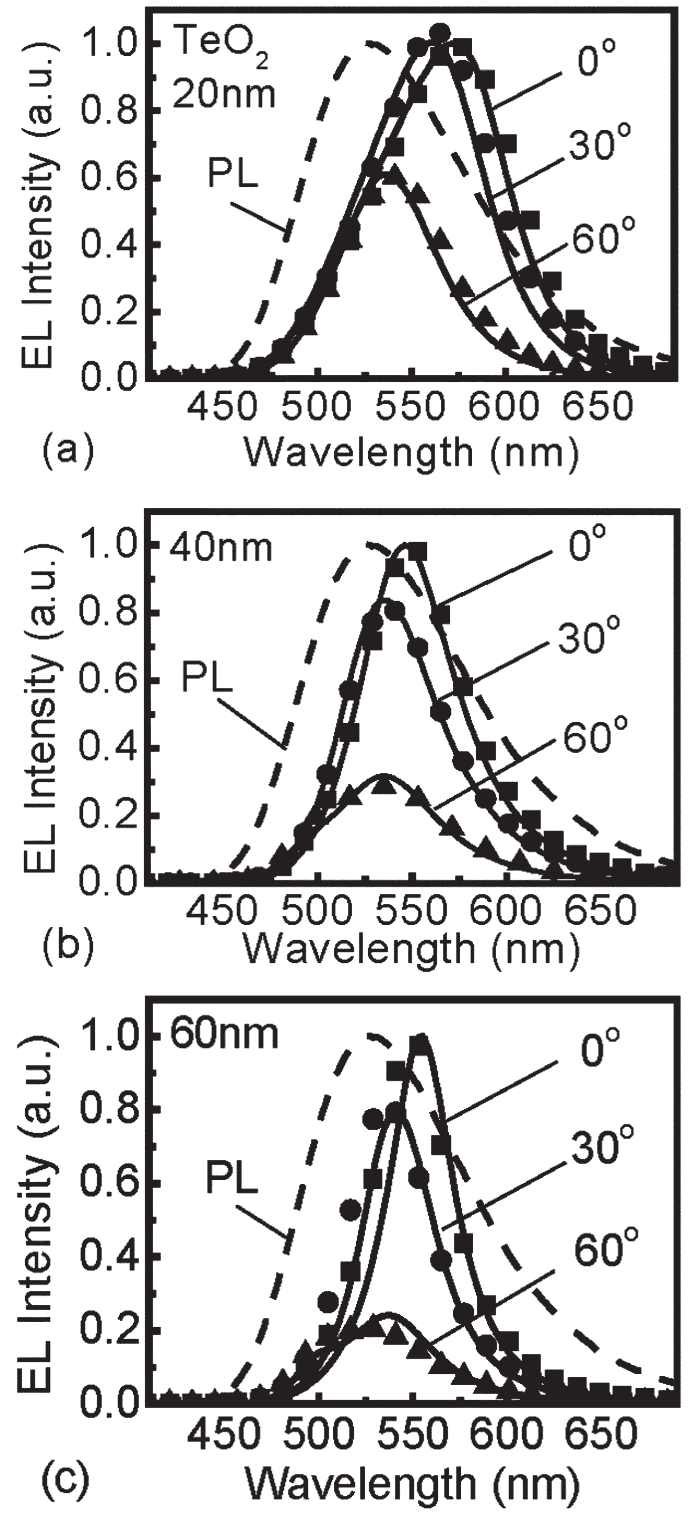

FIGURE 7 - Measured (solid symbols) and calculated (solid lines) EL spectra with relative intensities at viewing angles of $0^{\circ}, 30^{\circ}$, and $60^{\circ}$ for devices with a fixed organic structure of $\left(\mathrm{Alq}_{3}, m-\mathrm{MTDATA}\right)=(60$ and $30 \mathrm{~nm}$ ) and the varied $\mathrm{TeO}_{2}$ thickness of (a) $20 \mathrm{~nm}$, (b) $40 \mathrm{~nm}$, and (c) $60 \mathrm{~nm}$. In (a)-(c), the PL of $\mathrm{Alq}_{3}$ is also shown for comparison with EL spectra.

devices are similar to or slightly lower than that of a conventional bottom-emitting device (with an ITO anode), while a few previous reports have shown that top-emitting devices using high-reflectivity metal electrodes for both top and bottom electrodes can actually have higher efficiencies. $5,7,8$ However, one should notice that in these previous reports, $5,7,8$ $\mathrm{cd} / \mathrm{A}$ efficiencies, i.e., the on-axis emission, are examined, while in the present work, the total light out-coupling (the total external quantum efficiency) was examined. In an optimized microcavity OLED, due to redistribution of device emission more into the forward direction, one could obtain luminance (or cd/A efficiency) enhancement in the forward direction even without observing enhancement in the total external quantum efficiency. In our previous work, 
(a)
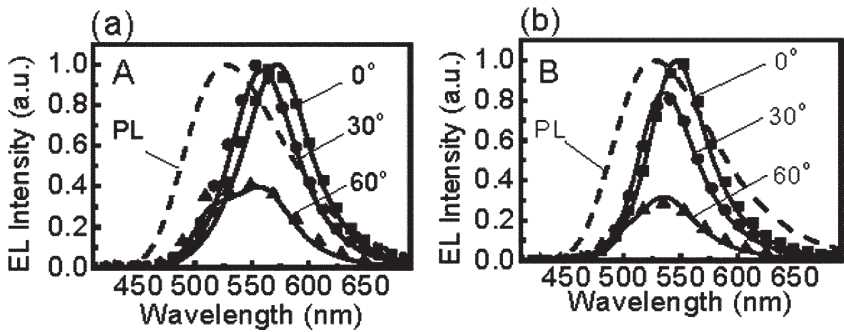

(c)

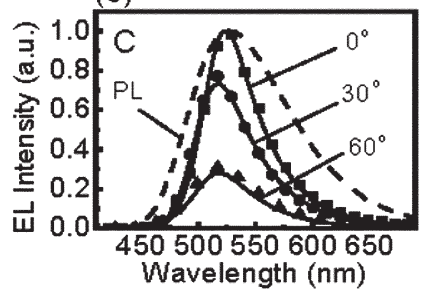

(d)

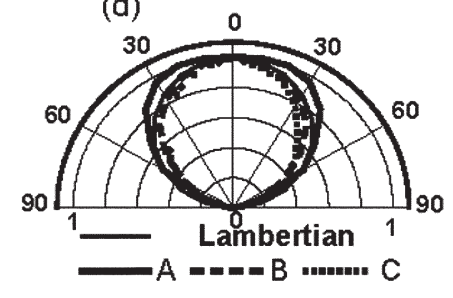

FIGURE 8 - Measured (solid symbols) and calculated (solid lines) EL spectra with relative intensities at viewing angles of $0^{\circ}, 30^{\circ}$, and $60^{\circ}$ for (a) device A, (b) device B, and (c) device C. (d) Polar plots of measured EL intensities for the three devices in comparison with the Lambertian distribution. In (a)-(c), the PL of $\mathrm{Alq}_{3}$ is also shown for comparison with EL spectra.

in top-emitting OLEDs with structures similar to the present device structure, substantial enhancement in cd/A efficiencies (i.e., on-axis emission) were indeed observed. ${ }^{8}$ In Figs. 8(a)-(c), again, one sees that the measured spectra and relative intensities (symbols) are in excellent agreement with calculated ones (solid lines). Roughly speaking, EL is red-shifted with increased cavity lengths and is blue-shifted with increased viewing angles, which are consistent with resonance conditions for an optical cavity. ${ }^{21}$ In the inset of Fig. 9, it is shown that the measured quantum efficiencies (symbols) also agree well with calculated out-coupling efficiencies (line). As expected from Fig. 4, device C gives the most saturated and stable green emission $v s$. viewing angles yet with tradeoffs in efficiency and Lambertian offset; device $\mathrm{B}$ gives an emission pattern closest to the ideal Lambertian distribution; and device A has the highest efficiency yet performs worst in other viewing characteristics. Indeed, emission of device A has shifted to yellow. Finally, one notices that with stronger microcavity effects, the emission characteristics of top-emitting OLEDs are very sensitive to layer thicknesses, which, in turn, would impose more stringent requirements on the thickness uniformity of deposition systems for large-area OLED displays.

\section{Conclusions}

In conclusion, we have analyzed emission characteristics of top-emitting OLEDs as a function of device structures using the rigorous classical electromagnetic model based on oscillating electric dipoles embedded in layered structures. From the comprehensive analysis, one extracts some clear trends in the dependence of various emission characteristics on device structures, based on which a general methodology for optimizing viewing characteristics of top-emitting

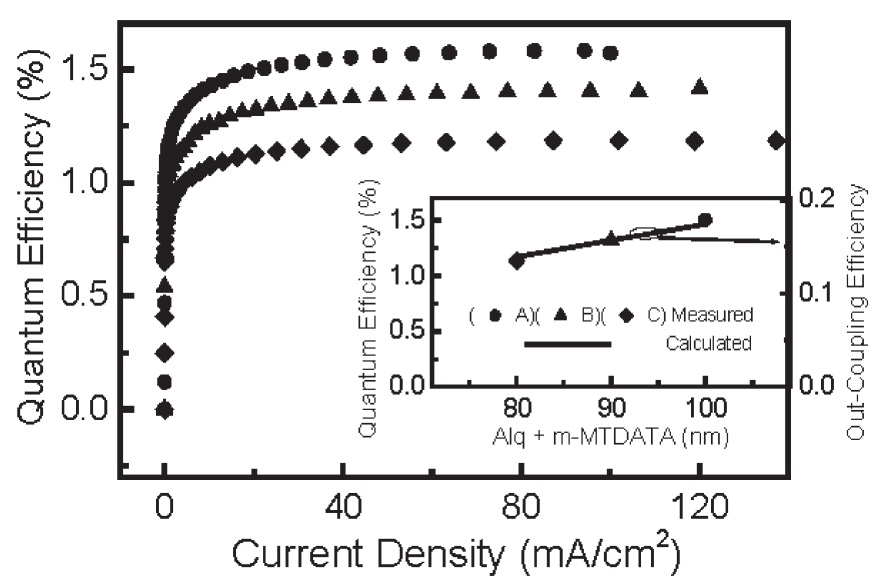

FIGURE 9 - Measured external EL quantum efficiencies of devices A, $B$, and C vs. driving current. Inset: comparison of EL efficiency (symbols) and calculated out-coupling efficiencies (line) of the three devices.

OLEDs for display applications is presented. Experimental results confirm the effectiveness of the analysis and the methodology.

\section{Acknowledgments}

The authors would like to acknowledge financial support from National Science Council (Grant No. NSC 92-2215-E002-023) of the Republic of China.

\section{References}

1 G W Jones, SID International Digest 32, 134 (2001).

2 T Sasaoka, M Sekiya, A Yumoto, J Yamada, T Hirano, Y Iwase, T Yamada, T Ishibashi, T Mori, M Asano, S Tamura, and T Urabe, SID International Digest 32, 384 (2001).

3 L S Hung, C W Tang, M G Mason, P Raychaudhuri, and J Madathil, Appl Phys Lett 78, 544 (2001).

$4 \mathrm{G} \mathrm{Gu}$, V Bulovic, P E Burrows, S R Forrest, and M E Thompson, Appl Phys Lett 68, 2006 (1996).

5 H Riel, S Karg, T Beierlein, B Ruhstaller, and W Rieß, Appl Phys Lett 82, 466 (2003).

6 M-H Lu, M S Weaver, T X Zhou, M Rothman, R C Kwong, M Hack, and J J Brown, Appl Phys Lett 81, 3921 (2002).

7 P K Raychaudhuri, J K Madathil, J D Shore, and S A. Van Slyke, J Soc Info Display 12, 315 (2003).

8 C-W Chen, P-Y Hsieh, H-H Chiang, C-L Lin, H-M Wu, and C-C Wu, Appl Phys Lett 83, 5127 (2003).

9 R H Jordan, L J Rothberg, A Dodabalapur, and R E Slusher, Appl Phys Lett 69, 1997 (1996).

10 N Takada, T Tsutsui, and S Saito, Appl Phys Lett 63, 2032 (1993).

11 Y Shirota, Y Kuwabara, H Inada, T Wakimoto, H Nakada, Y Yonemoto, S Kawami, and K Imai, Appl Phys Lett 65, 807 (1994).

12 J Shi and C W Tang, Appl Phys Lett 70, 1665 (1997).

13 W Lukosz, J Opt Soc Am 67, 1607 (1977).

14 J E Sipe, Surf Sci 105, 489 (1981).

15 K Neyts, J Opt Soc Am A 15, 962 (1998).

16 W L Barnes, J Mod Opt 47, 725 (2000).

17 C W Tang, S A Van Slyke, and C H Chen, Appl Phys Lett 65, 3610 (1989).

18 Color in Electronic Displays, edited by H. Widdel and D. L. Post (Plenum Press, New York, 1992).

19 C-W Chen, T-Y Cho, C-C Wu, H-L Yu, and T-Y Luh, Appl Phys Lett 81, 1570 (2002).

20 C-C Wu, C-W Chen, and T-Y Cho, Appl Phys Lett 83, 611 (2003).

$21 \mathrm{M}$ Born and E Wolf, Principles of Optics, 7th edn. (Cambridge University Press, Cambridge 1999). 


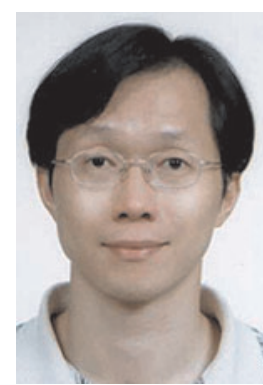

Chung-chih Wu received his B.S. degree in electrical engineering from National Taiwan University in 1990, and his M.A. and Ph.D. degrees in electrical engineering from Princeton University in 1994 and 1997, respectively. From 1997 to 1998, he was with the Electronic Research and Service Organization in the Industry Technology Research Institute (ERSO/ITRI), Hsin-Chu, Taiwan, as a researcher in the flat-panel-display division. In 1998, he joined the faculty of National Taiwan University in the Graduate Institute of Electro-optical Engineering and the Department of Electrical Engineering, where he is currently an associate professor. His current research interests include organic optoelectronic and electronic devices, and flat-panel displays. He is a member of the Society of Information Display (SID).

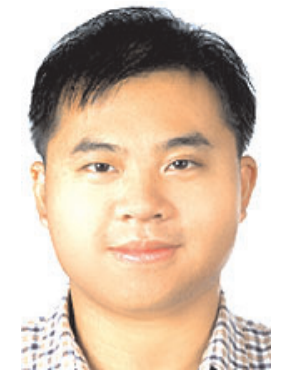

Chun-liang Lin received his B.S. degree in electrical engineering from National Taiwan University in 2001. He is currently a Ph.D. student in Graduate institute of Electro-Optical Engineering, National Taiwan University. His current research interests include organic optoelectronic and electronic devices, and flat-panel displays.

Ping-Yuan Hsieh received his B.S. degree in electrical engineering from the National Taiwan University in 2001. He received his M.S. degree from Graduate Institute of Electro-Optical Engineering, National Taiwan University in 2003

Huo-Hsien Chiang received his B.S. degree in physics from National Taiwan University in 2000. He received his M.S. degree from Graduate institute of Electro-Optical Engineering, National Taiwan University in 2002. 